\title{
Research to Market Transition of Mobile Assistive Technologies for People with Visual Impairments
}

\author{
Sergio Mascetti \\ Università degli Studi di Milano \\ Dipartimento di Informatica \\ sergio.mascetti@unimi.it
}

\author{
Dragan Ahmetovic \\ Università degli Studi di Torino \\ Dipartimento di Matematica \\ dragan.ahmetovic@unito.it
}

\author{
Cristian Bernareggi \\ Università degli Studi di Milano \\ Dipartimento di Informatica \\ cristian.bernareggi@unimi.it
}

\begin{abstract}
Mobile devices are accessible to people with visual impairments and hence they are convenient platforms to support assistive technologies. Indeed, in the last years many scientific contributions proposed assistive applications for mobile devices. However, few of these solutions were eventually delivered to end-users, depriving people with disabilities of important assistive tools. The underlying problem is that a number of challenges need to be faced for transitioning assistive mobile applications from research to market.

This contribution reports authors' experience in the academic research and successive distribution of three mobile assistive applications for people with visual impairment. As a general message, we describe the relevant characteristics of the target population, analyze different models of transition from academic research to end-users distribution and show how the transitioning process has a positive impact on research.
\end{abstract}

\section{Author Keywords}

Technology transfer; Mobile assistive technologies;

\section{CCS Concepts}

-Social and professional topics $\rightarrow$ Funding; Sustainability; Pricing and resource allocation; Software management;

\section{INTRODUCTION}

The last decade has witnessed a worldwide, ubiquitous diffusion of mobile devices. These devices are accessible and widely adopted also among people with visual impairments (VI). Indeed, most apps can be operated by people with VI using built-in accessibility functionalities such as magnifiers and screenreaders (VoiceOver on iOS and TalkBack on Android). Additionally, recent mobile devices feature computational and sensing capabilities (e.g., GPS, camera), that can be employed to develop innovative Assistive Technologies (AT). This favorable condition has led to the definition of mobile AT to support people with VI in different tasks such as orientation \& mobility [17, 20], education [1, 8] and entertainment [9].

\footnotetext{
Permission to make digital or hard copies of all or part of this work for personal or classroom use is granted without fee provided that copies are not made or distributed for profit or commercial advantage and that copies bear this notice and the full citation on the first page. Copyrights for components of this work owned by others than the author(s) must be honored. Abstracting with credit is permitted. To copy otherwise, or republish, to post on servers or to redistribute to lists, requires prior specific permission and/or a fee. Request permissions from permissions@ acm.org.

ASSETS '19, October 28-30, 2019, Pittsburgh, PA, USA

(C) 2019 Copyright held by the owner/author(s). Publication rights licensed to ACM. ISBN 978-1-4503-6676-2/19/10 . \$ \$15.00
}

DOI: http: //dx . doi .org/10.1145/3308561.3355618
Distributing innovative AT for people with VI entails significant research and development challenges. First, it is crucial to design and evaluate usable AT with people with VI across different contexts. In academic research this is achieved through user-centered design, prototype development and testing. Second, transitioning from research prototypes to large-scale distribution is a costly and demanding process, which is often disregarded in HCI research [5]. Therefore, the time-to-market of the latest scientific developments is often long and scientific advances remain frequently unavailable to end-users.

This contribution investigates the transition of research prototypes into final products in the field of mobile AT for people with VI. We report our experience, matured through academic research at Università degli studi di Milano and development at EveryWare Technologies, which was a spin-off of the same university. We describe development pipelines of three products, from the analysis conducted during research, to product distribution and maintenance. The objective was to deliver mobile AT to end-users with a sustainable process. Based on our experience we a) describe relevant characteristics of the target population and stakeholders, b) analyze different models for transitioning academic research to end-users and c) show how the transitioning process positively impacts research.

\section{RELATED WORK}

Technology transfer from research to market has been conducted in academia [27] and industry [24]. Indeed, many initiatives support the creation of startup and spin-off companies ${ }^{1}$, and strategies have been investigated to manage startups [23] and measure the impact of investments [10]. Nonetheless, innovative HCI ideas often fail to reach the market because they are only validated with a specific group of end-users, disregarding the role of other stakeholders [11]. To address this issue, prior research proposes a shift from user-centered to adoption-centered design [5], involving all stakeholders from the beginning of the research stage. Our experience confirms this finding and highlights additional obstacles for technology transfer of mobile AT for people with VI. First, there are often physical or cognitive differences among end-users (e.g., congenitally or late blind [12]). Hence additional effort is needed to design suitable interaction for different users. Second, misperception of AT may reduce their acceptance by end-users [25]. Third, AT discontinuance is high [22], and can affect their long-term permanence on the market.

\footnotetext{
${ }^{1}$ NSF I-CORPS:

https://www.nsf.gov/news/special_reports/i-corps/
} 


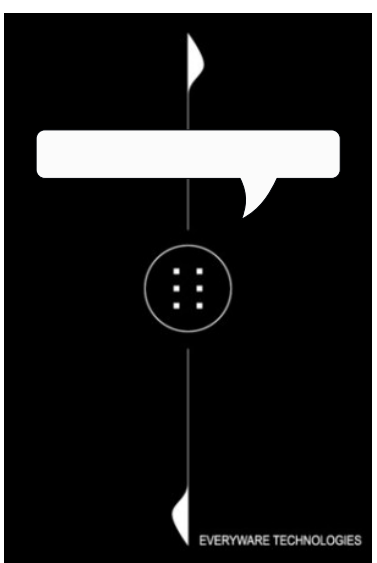

(a) TypeInBraille input screen

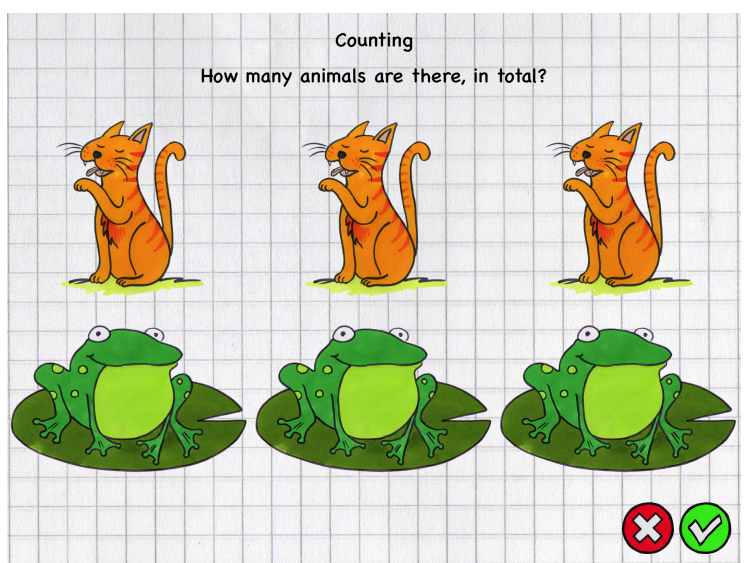

(b) MathMelodies counting exercise screen with audio icons

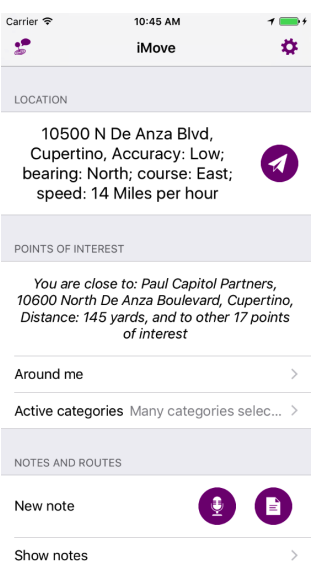

(c) iMove main view

Figure 1. Three mobile assistive apps developed and distributed by EveryWare Technologies

This contribution reports additional examples and insights about the outlined obstacles. In particular, it elaborates about population fragmentation, the importance of stakeholders in the dissemination process and suggests possible reasons for the high discontinuance rate.

\section{SUPPORTING TEXT ENTRY WITH TypeInBraille}

A major problem in the non-visual access on mobile devices relates to screenreader supported text entry using default onscreen keyboard [21]. TypeInBraille, shown in Figure 1(a), is an iOS app that substitutes keyboard text entry with a novel input method based on Braille ${ }^{2}$. Experimental results show that TypeInBraille enables faster and more accurate text entry [18]. This result is particularly relevant because the users involved in the tests were experienced with the on-screen keyboard, while they only trained for 10 minutes with TypeInBraille.

TypeInBraille was distributed through Business to Consumer (B2C) commercialization model: it was first developed using company resources and then marketed on the Apple App Store. The pricing policy was based on a market analysis and the price (\$4.99) was set in the same range as other similar AT apps. An advertisement campaign was conducted on blogs, thematic discussion groups, social media and online articles, before and in the months immediately following the app release. The communication with end-users followed a bi-directional model: informative and promotional messages were posted on social media, App store or company web site, at the same time end-users themselves inquired on how to use TypeInBraille in different scenarios, reported bugs and suggested extensions. End-users were very active both in starting and participating to new discussion threads and in contacting the development team directly from the TypeInBraille app.

The maintenance of TypeInBraille required a significant effort for bug fixing, preference adjustments, compliance with iOS updates and in particular for localization. Indeed, when the app was first released it was available in Italian and English only. However, after the app publication, users requested for

\footnotetext{
${ }^{2}$ Videos of TypeInBraille and other EveryWare Technologies apps: https: //www . youtube. com/user/EveryWareTech.
}

localization in other languages. The challenge in this case is that localization does not simply involve translating the app and instructions, but also adapting it to different Braille encodings $^{3}$. TypeInBraille was localized in other 4 languages thanks to a collaborative approach with final users: upon receiving a localization request, we would ask the user to translate the app and provide the correct encoding.

TypeInBraille was first released in October 2011 and it has since been downloaded by more than 1.000 users. Starting from version 8 (released in September 2014), iOS includes a native accessibility tool called "Braille Display Input" that allows users to insert text in Braille. Following the release of iOS 8, TypeInBraille reached its end-of-life stage and its maintenance has been discontinued.

\section{SUPPORTING MATH LEARNING WITH MathMelodies}

The teaching of STEM subjects is a known issue for people with VI [26]. Didactic tools for sighted students (especially for children) contain images and figures with the dual aim of capturing the student's attention and explaining concepts through their spatial representations (e.g., graphs and diagrams).

MathMelodies is a didactic software that supports primary school children in practicing Math [8, 4] through sophisticated proprioceptive interaction on mobile devices. The challenge is to design a software that is inclusive and engaging for both students with VI and sighted ones. The inclusiveness is achieved through multimodal interaction: the story can be either read, explored with a Braille display or accessed using screenreader. In particular, the interaction relies on "audio icons" [7], elements having a dual representation as images associated with characteristic sounds. Figure 1(b) shows an accessible counting exercise in which, upon touching an animal, its call is played. MathMelodies was designed with the help of teachers specialized in typhlology. User evaluations, conducted with sighted students and VI ones, show that all participants were independent and entertained during the interaction with the system, after a brief supervised training.

\footnotetext{
${ }^{3}$ Braille encompasses diverse encodings for different languages.
} 
To find a suitable business model for MathMelodies, we focused on the fact that the software has a clear social aim and it is easy to present to the public. Thus, we opted for crowdfunding [13] through a fixed-funding, donation-based campaign [2]. To warrant the success of the crowdfunding ${ }^{4}$ we engaged in an informative communication campaign on both traditional (radio, newspapers) and social media. A sample application, informative video clips and presentation documents localized in different languages were also used to advertise the crowdfunding. After the successful funding of the project, the first version of MathMelodies was engineered and published for free on the Apple App Store ${ }^{5}$. A communication campaign was initiated starting from donors involved in the funding stage. Since some of the donors were organizations for supporting people with VI they acted as marketing multipliers during the advertising effort. A follow-up crowdfunding campaign has successfully funded the second version of MathMelodies.

While the target funding goal was reached in both campaigns, the actual costs were higher than expected. In particular, we underestimated content creation (story writing, visual and audio design), and localization costs. The app still successfully reached the market because some of the professionals and organizations involved recognized the social value of the initiative and volunteered in the project.

\section{SUPPORTING ORIENTATION WITH iMove}

iMove (see Figure 1(c)) is a mobile AT for independent mobility of people with VI. It has the following key features:

- Provide on-demand information about user's position (e.g., current address) and nearby points of interest (POIs).

- Such information can also be notified periodically. The user can select which information to receive and how frequently, in terms of time and distance from the last notification.

- The user can create geo-referenced notes (i.e., an audio recording or a text), which are replayed when the user returns to the same location.

iMove development and distribution was sponsored by Retina Italia ONLUS ${ }^{6}$, an Italian non profit organization (NPO) that promotes education, employment and independent living of people with VI. The initial sponsorship covered the development costs and its distribution for free on the App Store for two years. The sponsorship was renewed to support the development of an updated app version and to keep it free for two additional years.

Concerning app engineering, no major challenge arose: the prototype developed as part of university research sketched the overall app design and no big implementation issues had to be faced. One non-technical challenge, which required some effort since our team did not have the required know-how, was the definition of legal documents: specifically privacy policy and the end-user license agreement. The first was needed to collect user consent for data acquisition and the second to define the limitation of liability in case of app malfunctions.

\footnotetext{
${ }^{4}$ https: //Www . indiegogo. com/projects/math-melodies

${ }^{5}$ https://itunes . apple. com/app/math-melodies/id713705958

$6_{\text {http: //www . retinaitalia.org/ }}$
}

The app was published ${ }^{7}$ in February 2013. By December 2018 it has been downloaded more than 300,000 times. From December 2015 iMove collects anonymous usage data including accessibility features enabled (e.g., VoiceOver, magnifier), app functionalities used and preferences settings. The analysis of this data shows that $44 \%$ of the users use accessibility tools and hence have a VI. These users use the app approximately four times more than others, and divide in four different user clusters $[15,14]$. We designed the app for users that want to be updated about their position while moving. We actually identified this user cluster, but it only accounted for $25 \%$ of the users. Others run iMove in short sessions to check the current address (20\%), the nearby POIs $(40 \%)$ or both $(15 \%)$. These results helped us designing two new functions specific for these users. One is the option to list nearby POIs on the main app screen. The other allows the users to be notified of the current address by hitting the device while in their pocket.

\section{LESSONS LEARNED AND DISCUSSION}

\section{Distributing $A T$ to a small and fragmented population}

Developing and distributing AT for people with VI helped us better understand the characteristics of the target population.

\section{Target population size}

World Health Organization estimates that there are 285 million of people with VI worldwide, a $4 \%$ of the total population ${ }^{8}$. Among these, 39 million ( $0.5 \%$ of world population) are blind. In industrialized countries, smartphone adoption is similar between people with VI [6] and others ${ }^{9}$. However, 90\% of people with VI live in low-income settings. Thus, we estimate that the target population for AT for people with VI is 2 to 3 orders of magnitude smaller than general mobile app users.

The size of the target population influences the whole pipeline of an AT project. Indeed, researchers struggle with finding user study participants, and commercializing AT may not be economically feasible in such a small market. One strategy to mitigate this issue is to pursue accessibility as one facet of a general purpose solution. For example, in one of our previous projects aimed at making a big exposition area in Milan accessible to people with VI, we realized that the proposed indoor positioning solution would be helpful to everyone and not only people with VI. Hence it was implemented in the customer's app for general public, with an inclusive user experience achieved by presenting navigation instructions both on a map for sighted users, and as text for screen-reader users.

\section{Population fragmentation due to difference in needs}

The target population is not homogeneous. Rather, it is segmented based on users' needs and capabilities. Often, the need for an AT is expressed only by a population segment, which further reduces the potential users. For example LightDetector $^{10}$, is an app that sonifies surrounding light intensity, which addresses the needs of blind users, but not low vision users.

\footnotetext{
${ }^{7}$ https://itunes . apple.com/app/imove/id593874954

${ }^{8} \mathrm{WHO}$ disability fact sheet:

http: //www . who . int/mediacentre/factsheets/fs282/en/

${ }^{9}$ Pew Research Center mobile fact sheet:

http: //www . pewinternet . org/fact-sheet/mobile/

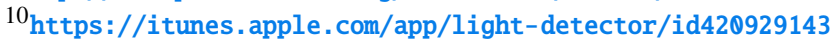


Similarly, the desired interaction paradigms are often different among population segments. For example, blind users require audio/haptic interaction, while low vision people generally prefer to use residual sight. Even among blind people relevant differences do exist. For example, congenitally blind people are more likely Braille readers than late blind people.

\section{User preferences}

Users' preferences and characteristics also influence the definition of the target user for a given application. For example, TypeInBraille is suitable for people with VI that own an iPhone, do not have enough residual sight to use the standard qwerty keyboard and are fluent with Braille. Indeed, the very small size of the target population is one of the reason of the relatively small diffusion of TypeInBraille. User preferences also influence how the users interact with the developed AT. For example, by analyzing iMove usage logs, we realized that different user's preferences result in different usage patterns.

\section{Preferred hardware and software platforms}

While Android devices can be cheaper and therefore open the distribution of assistive apps towards low-income settings, iOS devices have been historically more adopted by people with VI [19]. This claim also holds based on our experience: consider the LightDetector app for blind users, first developed for iOS devices and then ported to Android in 2015. During 22 months, between 2015 and 2017, the app was downloaded 73 times from Google Play and more than 2,100 times from Apple App Store. Based on our experience with MathMelodies (currently available for iPad only), we also point out that a relatively large number of potential users requested the app on a different platform (iPhone or an Android device). This, together with the small target population, may have also contributed for the relatively small diffusion of MathMelodies.

\section{Stakeholders}

Wile end-users are the main AT stakeholders, several others are also involved, such as caregivers (e.g., parents of people with VI), schools and teachers (especially those specialized in the education of students with VI), orientation and mobility specialists, associations and supporting organizations. In our experience, these stakeholders should also be involved during the development and distribution pipeline. For example our $i$ WalkStraight ${ }^{11}$ app was motivated by mobility instructors to let blind people practice walking straight. Another example is MathMelodies, that was developed thanks to the help of support teachers and typhlology experts [8]. The high social connectivity of the community of people with VI facilitates stakeholders involvement. Indeed there are many associations for people with VI that aggregate the target users and other stakeholders, and can help distributing the AT knowledge. Online social networking is also common, including online discussion groups (like VIPhone ${ }^{12}$ ), online AT directories ${ }^{13}$ and podcasts ${ }^{14}$. Leveraging the strong connectivity of the final users can help to reach more users, promote the product and reduce advertising costs.

\footnotetext{
${ }^{11}$ https://itunes . apple. com/app/iwalkstraight/id1137994131

${ }^{12}$ https://groups . google . com/forum/\#! forum/viphone

13 http: //wWw . teachingvisuallyimpaired.com/apps-for-vi .html

${ }^{14}$ http : //thatandroidshow . com/welcome-to-that-android-show/
}

\section{Challenges in research to market transition}

Based on our experience, the greatest challenge during transition from research to market is to find a suitable approach to sustain research, development and distribution. In particular the costs for engineering, publicizing and distributing a market-ready product do not provide additional research products and therefore are not eagerly addressed in academia. We review models for sustaining the process of distributing AT that we directly experimented or took into consideration.

\section{Commercialization to end-users}

The business to consumer (B2C) commercialization model is often used for AT (e.g., Sendero Seeing Eye GPS ${ }^{15}$ or Blindsquare ${ }^{16}$ ), and we followed it for TypeInBraille. One challenge with this approach is the relatively small market size and therefore limited economic potential, which would suggest that few competitors would venture into this field. Instead, there are several competitors (e.g., more than 20 outdoor orientation iOS apps for people with VI), which makes it even harder to create "blue oceans" of uncontested market space [16]. This could be one reason for the high discontinuance rate observed by Riemer et al [22]. Thus, a B2C approach can expose the project to risks such as long payback period, high maintenance costs and new competitors. In our experience with TypeInBraille this approach was sustainable only because initial costs were limited thanks to the availability of a prototype.

\section{Grant-funded distribution}

Grants are a common and often necessary mechanism to fund research in academia, but can also sponsor development and distribution end-user products. While we did not directly pursue this approach, we came across a number of AT funded through private or public grants which successfully reached end-users. One example is the VizWiz project [3], funded in part by US National Science Foundation. This approach reduces entrepreneurial risks since the funds are raised before the start of the project. However grant proposals generally follow constrained thematics, and therefore finding a suitable grant proposal and defining a project for it require significant effort and time.

\section{Crowdfunding}

Crowdfunding also acquires capitals before project start, thus reducing entrepreneurial risk. Additionally, running a crowdfunding campaign doubles as advertising and makes it possible to publish the app for free, which helps to reach a larger number of users. However, the crowdfunding campaign itself requires time, expertise and advertising, which in our experience resulted to be sustainable only in the short period. One of the main challenges is correctly determining the funding goal: for MathMelodies we kept the goal low, increasing the odds of successfully completing the campaign, but this resulted in a limited budget to cover the development costs. An interesting insight is that the majority of the funds do not come from people with VI or their caregivers. For this reason it is important to aim the communication not only to the target population or other stakeholders, but also to the general audience.

\footnotetext{
15 https://itunes . apple . com/app/seeing-eye-gps/id668624446

16 https://itunes. apple. com/it/app/blindsquare/id500557255
} 


\section{Sponsorship by private organizations}

Sponsorship is the strategy that worked better for EveryWare Technologies in delivering mobile AT to people with VI. As with crowdfunding and grant-funding, the funds are acquired before the project starts, thus minimizing the entrepreneurial risk. This approach also has a lower workload and time overhead since it does not require to wait for grant calls, write project proposals, or prepare crowdfunding campaigns. Instead, the main challenge is finding a sponsoring organization. We first approached big companies, aiming to address their corporate social responsibility. In our experience, however, innovative AT for people with VI were not appealing for such entities. Instead, we identified greater opportunities among NPOs committed to support independent living of people with VI that, in our experience, have limited resources, but are open to innovation and willing to support the distribution of AT.

\section{Non profit projects}

Public and private entities may also dedicate a part of their resources to the development and distribution of AT, with no direct economical benefit. This is often the case with tech companies, which often research AT and release them publicly at no cost to end-users. Mobile manufacturers and OS developers, influenced by third-party efforts, may also integrate popular AT on mobile platforms directly. For example, after a number of braille based input methods (including TypeInBraille) were released for iOs, Apple integrated their own braille input method in iOs $8^{17}$.

In academia, this approach is more difficult since developing and distributing market-ready AT does not directly benefit research. Partnerships between academic institutions and companies allow the former to focus on research aspects while the latter can support the engineering and development. One example of this approach is the NavCog project [20], an open source ${ }^{18}$ navigation assistant for people with VI. This project, researched jointly by the Cognitive Assistance Lab ${ }^{19}$ at Carnegie Mellon University and IBM-Research Tokyo, is provided at no cost to end-users ${ }^{20}$.

\section{Advertising}

One common revenue model adopted in web and mobile applications distribution is based on advertising. This model has two main drawbacks in the field of AT. First, to generate significant revenue a large number of users is needed. Instead the target population is relatively small. Second, advertisements are often visual elements that not only cannot be accessed by people with VI, but they can also hinder app accessibility.

There are two additional differences with respect to the sponsorship model: 1) In sponsorship, the budget is defined before the development starts, while in the advertising model revenues are only obtained once the app is published, 2) In the sponsorship model there are no obtrusive advertisements, save for minimal sponsor acknowledgement.

\footnotetext{
${ }^{17}$ Apple Vision native assistive technologies:

https://www . apple. com/accessibility/iphone/vision/

18 https: //github. com/hulop

19 http: //WwW . cs . cmu . edu/ NavCog/

20 https: //itunes . apple. com/app/navcog/id1042163426
}

\section{How transition to market benefits research}

Why should academic researchers be interested in distributing AT? An important driving force is that the accessibility research, that would otherwise remain confined to academic publications, is able to reach and assist individuals with disabilities in solving everyday tasks. Personal satisfaction is also a motivator for recruiting volunteer staff, which can help to reduce development and distribution costs. AT commercialization can also positively impact research activities along the following two main directions.

\section{Remote data collection.}

The majority of research studies in the field of AT are validated through short evaluation sessions involving few participants in a controlled environment. Remote collection of usage data allows researchers to overcome these limitations, thus paving the way to longitudinal studies with thousands of participants in real-world scenarios. We adopted this approach to analyze usage patterns of iMove users. We collected 15 months of usage data from more than 61,000 users, discovering user accessibility preferences (VoiceOver, magnifiers, inverted colors, etc..) and relevant orientation information. Such results are helpful for better understanding user behavior, technology adoption and for designing other AT.

\section{Know-how.}

Know-how sharing between academia and industry is mutually beneficial. Indeed, between EveryWare Technologies and the affiliated university laboratory, there was a tight collaboration and frequent overlap between research team members and professionals involved. EveryWare Technologies benefited from the research team's knowledge of the state of art and novel technical advancements, while researchers acquired engineering and development experience through activities conducted for EveryWare Technologies. This speeds up the development of research prototypes, hence boosting the research Also, distributing apps requires to know the current state of the market and competing solutions, which is a useful knowledge for defining new research directions.

\section{CONCLUSIONS}

Every year several AT for people with VI are researched and evaluated. Still, few of these are ultimately brought to market. One reason is the small size of the target population, which makes it hard to create a sustainable transitioning process under general market rules. When planning to transform a research prototype into a finite product it is therefore necessary to carefully select the most suitable approach to finance the transitioning process, also given the characteristics and the size of the specific fragment of the target population. We describe three approaches for transitioning research prototypes to market in the field of mobile AT that we experimented and we identify their peculiar characteristics which should be taken into consideration during this process. Finally, we identify positive effects that the transitioning process has on research, which complete the loop: research feeds apps distribution, which in turns boosts research. Under this perspective the transitioning process makes sense also from the point of view of academia: the effort to publish an app is justified by the impact that this process has on research. 


\section{REFERENCES}

[1] Dragan Ahmetovic, Cristian Bernareggi, João Guerreiro, Sergio Mascetti, and Anna Capietto. 2019.

AudioFunctions.web: Multimodal Exploration of Mathematical Function Graphs. In Web For All C. ACM.

[2] Paul Belleflamme, Nessrine Omrani, and Martin Peitz. 2015. The economics of crowdfunding platforms. Information Economics and Policy.

[3] Jeffrey P Bigham, Chandrika Jayant, Hanjie Ji, Greg Little, Andrew Miller, Robert C Miller, Robin Miller, Aubrey Tatarowicz, Brandyn White, Samual White, and others. 2010. VizWiz: nearly real-time answers to visual questions. In S. on UI software and technology. ACM.

[4] Niccolò Cantù, Mattia Ducci, Dragan Ahmetovic, Cristian Bernareggi, and Sergio Mascetti. 2018. MathMelodies 2: a Mobile Assistive Application for People with Visual Impairments Developed with React Native. In C. on Computers and Accessibility. ACM.

[5] Parmit K. Chilana, Andrew J. Ko, and Jacob Wobbrock. 2015. From User-Centered to Adoption-Centered Design: A Case Study of an HCI Research Innovation Becoming a Product. In C. on Human Factors in Computing Systems. ACM.

[6] Michael D. Crossland, Rui S. Silva, and Antonio F. Macedo. 2014. Smartphone, tablet computer and e-reader use by people with vision impairment.

[7] William W Gaver. 1986. Auditory icons: Using sound in computer interfaces. Human-computer interaction.

[8] Andrea Gerino, Nicolò Alabastro, Cristian Bernareggi, Dragan Ahmetovic, and Sergio Mascetti. 2014. Mathmelodies: inclusive design of a didactic game to practice mathematics. In Int. C. on Computers Helping People with Special Needs. Springer.

[9] Andrea Gerino, Lorenzo Picinali, Cristian Bernareggi, and Sergio Mascetti. 2015. Eyes-free exploration of shapes with invisible puzzle. In $C$. on Computers \& Accessibility. ACM.

[10] Gunnar Glänzel and Thomas Scheuerle. 2016. Social impact investing in Germany: Current impediments from investors' and social entrepreneurs' perspectives. VOLUNTAS: Int. J. of Voluntary and Nonprofit Org. .

[11] Austin Henderson. 2005. The innovation pipeline: design collaborations between research and development. Interactions.

[12] Marion Hersh and Michael A Johnson. 2008. Assistive Technology for Visually Impaired and Blind People. Springer.

[13] Christopher Michael Homan, Jon I Schull, and Akshai Prabhu. 2017. On the Genesis of an Assistive Technology Crowdsourcing Community. In Ext. Abst. on Human Factors in Computing Systems. ACM.

[14] Hernisa Kacorri, Sergio Mascetti, Andrea Gerino, Dragan Ahmetovic, Valeria Alampi, Hironobu Takagi, and Chieko Asakawa. 2018. Insights on Assistive
Orientation and Mobility of People with Visual Impairment Based on Large-Scale Longitudinal Data. $T$. on Accessible Comp..

[15] Hernisa Kacorri, Sergio Mascetti, Andrea Gerino, Dragan Ahmetovic, Hironobu Takagi, and Chieko Asakawa. 2016. Supporting Orientation of People with Visual Impairment: Analysis of Large Scale Usage Data. In C. on Computers and Accessibility. ACM.

[16] W Chan Kim and Renee A Mauborgne. 2014. Blue ocean strategy. Harvard Press.

[17] Sergio Mascetti, Dragan Ahmetovic, Andrea Gerino, and Cristian Bernareggi. 2016. Zebrarecognizer: Pedestrian crossing recognition for people with visual impairment or blindness. Pattern Recognition.

[18] Sergio Mascetti, Cristian Bernareggi, and Matteo Belotti. 2012. TypeInBraille: quick eyes-free typing on smartphones. In Int. C. on Computers Helping People with Special Needs. Springer.

[19] John Morris and James Mueller. 2014. Blind and deaf consumer preferences for android and iOS smartphones. In Inclusive Designing. Springer.

[20] Masayuki Murata, Dragan Ahmetovic, Daisuke Sato, Hironobu Takagi, Kris M Kitani, and Chieko Asakawa. 2019. Smartphone-based localization for blind navigation in building-scale indoor environments. Pervasive and Mobile Comp..

[21] Hugo Nicolau, Kyle Montague, Tiago Guerreiro, André Rodrigues, and Vicki L Hanson. 2017. Investigating Laboratory and Everyday Typing Performance of Blind Users. T. on Accessible Comp..

[22] Marti L Riemer-Reiss and Robbyn R Wacker. 2000. Factors associated with assistive technology discontinuance among individuals with disabilities. $J$. of Rehabilitation.

[23] Eric Ries. 2011. The Lean Startup: How Today's Entrepreneurs Use Continuous Innovation to Create Radically Successful Businesses. Crown Business.

[24] Curt J. Sampson. 2009. Experience Report: Haskell in the 'Real World': Writing a Commercial Application in a Lazy Functional Lanuage.

[25] Kristen Shinohara and Jacob O Wobbrock. 2011. In the shadow of misperception: assistive technology use and social interactions. In C. on Human Factors in Computing Systems. ACM.

[26] Paul Stanley. 2008. Assessing the mathematics related communication requirements of the blind in education and career. In Int. C. on Computers for Handicapped Persons. Springer.

[27] Randy Thompson. 2012. Why Tenure is Not a Private Sector Term: A Look at the Role of Educational Institutions in a Changing World of Commercialization, Investing, and Community Development. In C. on Information Technology Education. ACM. 